\title{
PUNCTUAL HILBERT SCHEMES ${ }^{1}$
}

\author{
BY A. IARROBINO ${ }^{2}$
}

Communicated by Michael Artin, March 23, 1972

This note is about the structure of families of open ideals in the ring of power series in two variables. The Hilbert scheme parametrizing them is stratified into locally closed subschemes $Z_{T}$, whose dimension we calculate. We then discuss some global consequences for families of 0-dimensional schemes on a surface ( $\$ 1$, Corollaries 2, 3). Except in low characteristics, $Z_{T}$ is locally an affine space (Theorem 2) and is a locally trivial bundle over the complete variety $G_{T}$ parametrizing graded ideals of type $T$ (Theorem 3 ).

1. A stratification of the Hilbert scheme. Let $R$ be the ring of power series $k\left[\left[x_{1}, \ldots, x_{r}\right]\right]$ in $r$ variables over an algebraically closed field $k$, with maximal ideal $m$; and let $R_{j}$ denote the space of forms of degree $j$ in $R$, so that $R=\prod R_{j}, j=0, \ldots, \infty$. If $I$ is an ideal in $R$, we let $I_{j}$ denote the space of forms in $R_{j}$ which are initial forms of elements of $I$. By the type of $I$ we mean the sequence

$$
T(I)=\left(t_{0}, t_{1}, \ldots, t_{j}, \ldots\right), \quad \text { where } t_{j}=\operatorname{dim}_{k}\left(R_{j} / I_{j}\right) .
$$

We will sometimes refer to a type $T$, meaning a specific infinite sequence $\left(t_{0}, t_{1}, \ldots\right)$. By the length $|T|$ of $T$ we mean $\sum t_{j}$, if it is finite. The initial degree of $I$ is the smallest $j$ for which $I_{j} \neq 0$. It depends only on the type of $I$. It is easy to show that if $I$ has finite colength $n$, then $n=|T(I)|$, and $t_{j}=0$ if $j \geqq n$.

Let Hilb $R$ be the Hilbert scheme parametrizing the family of ideals of colength $n$ in $R$, and $Z_{T}$ the subscheme parametrizing ideals of a given type $T$ where $|T|=n$. Then we get a stratification (see [7])

$$
\operatorname{Hilb}^{n} R=\underset{|T|=n}{\bigcup} Z_{T} .
$$

For the rest of the paper we consider the case $r=2$, and let $A=k[[x, y]]$. If $I \subset A$ has colength $n$ and initial degree $d$, then

AMS 1970 subject classifications. Primary 14C05; Secondary 14M15, 14F05, 13J05, $14 \mathrm{C} 25,14 \mathrm{~N} 10$.

Key words and phrases. Hilbert schemes, 0-cycles, irreducibility, Chow morphisms, surfaces, locally trivial bundles, power series rings, families of ideals.

${ }_{1}^{1}$ Supported in part by NSF Grant GP-29028.

${ }^{2}$ Most of the results announced comprise part of the author's Ph.D. thesis, written under the direction of A. Mattuck at M.I.T. in 1970. 


$$
\begin{aligned}
T(I) & =\left(1,2, \ldots, d, t_{d}, \ldots, t_{n-1}, 0,0, \ldots\right), \\
0 & \leqq t_{j} \leqq j+1, \sum t_{j}=n \text { and } d \geqq t_{d} \geqq \ldots \geqq t_{n-1} .
\end{aligned}
$$

The above conditions (2) characterize the sequences that occur as types of an open ideal in $A$. We assume $T$ is a sequence satisfying (2), and set

$$
\begin{aligned}
e_{j} & =t_{j-1}-t_{j} & & \text { if } j \geqq d, \\
& =0 & & \text { otherwise. }
\end{aligned}
$$

We call $e_{j}$ the jump index of $T$. We let $G_{T}$ be the subscheme of Hilb ${ }^{n} R$ parametrizing the graded ideals of type $T$.

THEOREM 1.

$$
\begin{gathered}
\operatorname{dim} Z_{T}=n-\sum e_{j}\left(e_{j}+1\right) / 2=n-d-\sum e_{j}\left(e_{j}-1\right) / 2, \\
\operatorname{dim} G_{T}=\sum_{j \geqq d}\left(e_{j}+1\right) e_{j+1} .
\end{gathered}
$$

The proof is by an induction on the colength $n$. We compare ideals $I$ of type $T$ having a certain weak normal form with the ideals $I: x$, which have a type $T: x$ which depends only on $T$ and is of length $<n$.

Let $T_{n}=(1,1, \ldots, 1,0,0, \ldots)$, where $\left|T_{n}\right|=n$ and $n>1$.

\section{COROLlary 1.}

$$
\begin{aligned}
\operatorname{dim} Z_{T} & =n-1=\operatorname{dim} \operatorname{Hilb}^{n} A & & \text { if } T=T_{n}, \\
& <n-1 & & \text { if } T \neq T_{n},|T|=n .
\end{aligned}
$$

Proof. If $d=1$, then $T=T_{n}$ by (2) and $\operatorname{dim} Z_{T}=n-1$ by (4). If $d>1$ then $\operatorname{dim} Z_{T} \leqq n-d \leqq n-2$.

Suppose $X$ is a nonsingular surface, and consider the Chow morphism (see [2]) onto the $n$-fold symmetric product $X^{(n)}$ :

$$
w_{n}: \operatorname{Hilb}^{n} X \rightarrow X^{(n)}
$$

Hilb $^{n} X$ is a desingularization of $X^{(n)}$. If $z$ is a geometric point of $X^{(n)}$ representing the zero-cycle $W=\Sigma n_{i} Q_{i}$, of degree $n$ on $X$, then $w_{n}^{-1}(z)$ $\simeq \prod$ Hilb $^{n_{i}} A$ and $w_{n}^{-1}(z)$ parametrizes the subschemes of $X$ having cycle $W($ see $[2])$. This shows

Corollary 2. $\operatorname{dim} w_{n}^{-1}(z)=\sum_{i=1}^{r}\left(n_{i}-1\right)=n-r$.

Let $\pi$ denote the partition $\left(n_{1}, \ldots, n_{r}\right)$ of $n$, and let $X_{\pi}$ be the subvariety of $X^{(n)}$ parametrizing cycles of index $\pi$, and $Y_{n}=w_{n}^{-1}\left(X_{n}\right)$. Then by Corollary 2, 


$$
\operatorname{dim} Y_{\pi}=n+r \quad \text { and } \operatorname{cod} Y_{\pi}=n-r .
$$

Let $D$ denote the singular locus on $X^{(n)}$, parametrizing cycles $\Sigma n_{i} Q_{i}$ where some $n_{i} \neq 1$, and let $B=w_{n}^{-1}(D)$. $B$ is the branch locus of the universal subscheme $Z^{n}$,

$$
X \times \operatorname{Hilb}^{n} X \supset Z^{n} \rightarrow \operatorname{Hilb}^{n} X,
$$

over $\mathrm{Hilb}^{n} X$ (see [2] or [1]).

COROllary 3. B is irreducible.

Proof. By (5), the highest dimensional component of $B$ is just $Y_{[2]} \bmod$ a lower dimensional subvariety, where [2] denotes the partition $(2,1,1, \ldots)$ of $n$. But $X_{[2]}$ is irreducible, and the fibers of $w_{n}: Y_{[2]} \rightarrow X_{[2]}$ are projective lines, so $Y_{[2]}$ is irreducible. $Z^{n}$ is flat over the nonsingular $\mathrm{Hilb}^{n} X$, so it is Cohen-Macauley. It must be nonsingular in codimension 1 , since any singularity would have to be over $Y_{[2]}$ and we can reduce to the case Hilb $^{2} X$ where $Z^{2}$ is nonsingular. Therefore $Z^{n}$ is normal, and by purity of branch locus, $B$ is pure codimension 1 . Corollary 3 follows from the irreducibility of $Y_{[2]}$.

From Corollary 3 one deduces that $\operatorname{Pic}\left(\operatorname{Hilb}^{n} X\right) \otimes Q=Q \oplus \operatorname{Pic} X^{(n)} \otimes Q$.

2. The varieties $Z_{T}$ and $G_{T}, r=2$. We assume $|T|=n$. Except where noted, the results are valid in all characteristics.

THEOREM 2. $Z_{T}$ and $G_{T}$ each have a connected cover by Zariski opens in an affine space, hence they are irreducible, rational, and nonsingular. $G_{T}$ is also complete.

THEOREM $2^{\prime}$. If char $k=0$, or char $k>n, Z_{T}$ and $G_{T}$ each have a connected cover by open sets isomorphic to affine spaces.

The proof uses a normal form for ideals of type $T$ in $A$. Theorem $2^{\prime}$ arises from a better normal form in those cases.

To each ideal $I$ we associate the completed graded ideal $\operatorname{gr}(I)=\prod I_{j}$, and clearly $T(I)=T(\mathrm{gr} I)$. This leads to a morphism $\pi: Z_{T} \rightarrow G_{T}$, having a section $s: G_{T} \rightarrow Z_{T}$ induced by the inclusion of graded ideals of type $T$ in all ideals of type $T$.

THEOREM 3. $\pi: Z_{T} \rightarrow G_{T}$ is a locally trivial bundle having fibre an affine space and having a natural "0-section" $s$. In general, $Z_{T}$ is not an algebraic vector bundle over $G_{T}$.

These are bundles with group $\operatorname{Aut}(\boldsymbol{A})$, where $\boldsymbol{A}=$ affine space, but whose group cannot in general be reduced to $G l(A)$. In characteristic 0 , such a bundle is diffeomorphic (but not algebraically isomorphic) to a vector bundle. See the example below. 
The structure of $G_{T}$ is known only in the simplest cases; for example, if $T$ satisfies $e_{j}\left(e_{j+1}\right)=0$ for all $j$, then $G_{T}=\prod_{j>d} P_{e_{j}}$.

ExAmple. If $T=T_{n}$, then $Z_{T}$ parametrizes ideals in $A$ of colength $n$, and initial degree 1 . Typical such ideals are

$$
I_{C}=\left(y+c_{0} x+\ldots+c_{n-2} x^{n-1}, m^{n}\right)
$$

and

$$
I_{B}=\left(x+b_{0} y+\ldots+b_{n-2} y^{n-1}, m^{n}\right) .
$$

Thus $\operatorname{gr}\left(I_{C}\right)=\left(y+c_{0} x, m^{n}\right)$, and $\operatorname{gr}\left(I_{B}\right)=\left(x+b_{0} y, m^{n}\right)$.

Every ideal of type $T_{n}$ has one or both of the above forms; $c_{0}$ and $b_{0}$ are coordinates on the two affine pieces of $P_{1}=G_{T}$; and the analysis of $Z_{n}$ proceeds from an analysis of the transition functions:

$$
I_{B}=I_{C} \Leftrightarrow\left\{\begin{array}{c}
b_{0}=g_{0}\left(c_{0}^{-1}\right)=c_{0}^{-1} \\
b_{j}=g_{j}\left(c_{0}^{-1}, c_{1}, \ldots, c_{j}\right) \\
\ldots
\end{array}\right\} .
$$

There is a natural projection from ideals of type $T_{n}$ to ideals of type $T_{n-1}: I \rightarrow I+m^{n-1}$ or "ignore the last coefficient," hence a projection $p_{n}: Z_{n} \rightarrow Z_{n-1}$, and there is an exact sequence of bundles over $P_{1}$,

$$
0 \rightarrow \mathcal{O}(n) \rightarrow Z_{n} \stackrel{p_{n}}{\rightarrow} Z_{n-1} \rightarrow 0,
$$

exact in the sense that $\mathcal{O}(n)=p_{n}^{-1}\left(s\left(P_{1}\right)\right)$.

By (6), $Z_{3}=\mathcal{O}(3)$ over $P_{1}$. However in [8] we show that if $n>3$, then $Z_{n}$ is not a vector bundle.

The group of substitutions with determinant one, $\left(\operatorname{Aut}\left(A / m^{n}\right)\right)$, acts on ideals in $A / m^{n}$ and preserves the type, so $\operatorname{Aut}\left(A / m^{n}\right)$ acts on $Z_{T}$ if $|T| \leqq n$. The action is clearly transitive on $Z_{n}$, and the isotropy group of the point $p \in Z_{n}$ corresponding to $I=\left(x, m^{n}\right)$ is the triangular subgroup $\Delta_{n}$ of substitutions $x \rightarrow u^{-1} x, y \rightarrow u y+a x$ with $u \in\left(A / m^{n}\right)^{*}$ and $a \in\left(A / m^{n-1}\right)$. Thus $Z_{n}=\operatorname{Aut}\left(A / m^{n}\right) / \Delta_{n}$, a quotient variety for the left cosets of $\Delta_{n}$. This example generalizes to $r>2, G_{T}=P_{r}$.

In general $S l_{2}(k)=S l_{2}(A / m)$ acts on $G_{T}$, and the kernel $K$,

$$
1 \rightarrow K \rightarrow \operatorname{Aut}\left(A / m^{n}\right) \rightarrow S l_{2}(A / m) \rightarrow 1,
$$

acts on the fibre of $Z_{T}$ over $G_{T}$ if $|T|=n$, and neither action is transitive. The analysis of this action will certainly lead to deeper results.

The dimension results of $\$ 1$ and the irreducibility of $Z_{T}$ given in Theorem 2 support the long standing

CONJECTURE 1 . Hilb ${ }^{n} A$ is irreducible, $\mathrm{Hilb}^{n} A=\bar{Z}_{n}$, the completion of $Z_{n}$. 
This conjecture is implied by

CONJECTURE 2. $Z_{T}$ is in the singular locus of $\mathrm{Hilb}^{n} A$ if $T \neq T_{n}$.

We have checked Conjecture 2 if $T$ has initial degree 2 and in some other cases - in fact all cases where we have specific parameters for ideals near a given ideal $I$ of colength $n$ in $A$. The analogous conjectures are false in higher dimensions [9].

\section{BIBLIOGRAPHY}

1. B. Bennett, On the characteristic functions of a local ring, Ann. of Math. (2) 91 (1970), 25-87. MR $40 \# 5608$.

2. J. Fogarty, Algebraic families on an algebraic surface, Amer. J. Math. 90 (1968), 511-521. MR 38 \#5778.

3. - Truncated Hilbert functors, J. Reine Angew. Math. 234 (1969), 65-88. MR 39 \#5585.

4. - The punctual Hilbert schemes of an algebraic surface (to appear).

5. A. Grothendieck, Fondements de la géométrie algébrique, [Extraits du Séminaire Bourbaki, 1957-1962], Secrétariat mathématique, Paris, 1962. MR 26 \#3566.

6. R. Hartshorne, Connectedness of the Hilbert scheme, Inst. Hautes Etudes Sci. Publ. Math. No. 29 (1966), 5-48. MR 35 \#4232.

7. A. Iarrobino, Families of ideals in the ring of power series in two variables, Thesis, M.I.T., Cambridge, Mass., 1970 (to appear).

8. - Families of linear ideals in $k\left[\left[x_{1}, \ldots, x_{r}\right]\right]$ : Some locally trivial bundles that are not vector bundles, over $P_{r}$ and $\mathrm{Grass}_{r}^{s}$ (to appear).

9. - Reducibility of the families of 0-dimensional schemes on a variety, Invent. Math. 15 (1972), 72-77.

10. - Bundles over $P_{1}$ with fibre an affine plane (to appear).

Department of Mathematics, University of TeXas, Austin, TeXas 78712

Current address: Department of Mathematics, University of California, Berkeley, CALIFORNIA 94720 